\title{
Stability of $p$-Harmonic Maps
}

\author{
Tadashi NAGANO and Makiko SUMI
}

\author{
Sophia University
}

\section{Introduction.}

We will study stability of $p$-harmonic maps (1.3; called harmonic if $p=2)$ of compact Riemannian manifolds into others when those maps are isometric and totally geodesic and prove that they are stable for sufficiently large $p$ if and only if they are stable as minimal immersions (Theorem 2.12). The proof is not hard but the theorem applies to numerous important examples, (3.1) through (3.10), to establish stability for large $p$; in all of them, the maps are stable minimal immersions of compact symmetric spaces into other ones. As to the number $p$, a sharp estimate is given in case the domain of the map is Einsteinian (Theorem 2.14).

Our motivation is this. If one wants to know the structure of the space $C^{\infty}(M, N)$ of the smooth maps of a compact manifold $M$ into another, then one would like to see the Palais-Smale condition (C) and regularity both valid in an appropriate completion of $C^{\infty}(M, N)$. The $p$-energy seems to be a most promising function (See [U]).

We thank Prof. Y. Ohnita for invaluable advices. For one, after we had submitted this paper to the Journal, he showed us a copy of a preprint by A. El Soufi \& A. Jeune.

\section{Preliminaries.}

Given a smooth map ( $C^{\infty}$-map) $f: M \rightarrow N$ of a compact connected Riemannian manifold $M$ into another, we denote the p-energy of $f$ by

$$
E=E_{p}=E_{p}(f):=p^{-1} \int_{M}\|d f\|^{p} \omega,
$$

where $p$ is a real with $p \geqq 2$ (for simplicity) and $\omega$ is the volume element. The length $\|d f\|$ of the differential of $f$ is defined in the natural fashion; $\|d f\|^{2}=\langle d f, d f\rangle$. Computation of its first variation yields the Euler-Lagrange equation

\section{2 .}

$$
\nabla^{*}\left(\|d f\|^{p-2} d f\right)=0
$$


out of $\left(\|d f\|^{p-2} d f, \nabla v\right)=0$ for a smooth homotopy $f_{t},|t|<\varepsilon$, of $f=f_{0}$, where $v:=\left.\partial_{t}\right|_{t=0} f_{t}$, the parentheses $($,$) denote the L^{2}$ inner product and $\nabla^{*}$ is the formal adjoint of $d$ or that of the covariant derivation $\nabla$ for the sections of the pull back $f^{*} T N$ of the tangent bundle $T N$ with the metric and the associated bundles. We will write $E$ for $f^{*} T N$.

1.3. Definition. The $C^{\infty}$-map $f$ is $p$-harmonic iff it is a solution of the EulerLagrange equation 1.2.

1.4. A $p$-harmonic map $f$ may be, in terms of local coordinates, characterized (as in [EL] for $p=2$ ) by

$$
\text { 1.5. } \quad g^{i j} \partial_{i}\left(\|d f\|^{p-2}\right) \partial_{j} f^{q}+\|d f\|^{p-2} g^{i j}\left(\partial_{i} \partial_{j} f^{q}-{ }^{M} \Gamma_{i j}^{k} \partial_{k} f^{q}+{ }^{N} \Gamma_{r s}^{q} \partial_{i} f^{r} \partial_{j} f^{s}\right)=0,
$$

where $g$ is the Riemannian metric of $M$ with the Christoffel symbol ${ }^{M} \Gamma$ and similarly for ${ }^{N} \Gamma$. One readily sees it, since one has $\nabla^{*} \rho=-\operatorname{Tr} \nabla \rho$ for an $E$-valued 1-form $\rho, \operatorname{Tr}$ denoting the trace.

Here are a few observations about the Euler-Lagrange equation.

1.6. In case $f$ is an isometric immersion, $f$ is harmonic (i.e. 2-harmonic) if and only if $f$ is $p$-harmonic for every $p \geqq 2$. In fact $\|d f\|^{2}$ is then a constant $(=\operatorname{dim} M)$.

1.7. The map $f$ is $p$-harmonic for every $p \geqq 2$ if and only if (1) $f$ is harmonic and (2) $d f\left(\operatorname{grad}\|d f\|^{2}\right)=0$. Notice that (2) just states the vanishing of the first term in the right hand side of (1.5).

Now we proceed to describe the Jacobi operator $J=J_{p}$ and the second variation formula. Let $\left(f_{s, t}\right)$ be a two-parameter variation of the $p$-harmonic map $f=f_{0,0}$, $|s|,|t|<\varepsilon$. Then a straightforward calculation gives the second variation formula

1.8 .

$$
\left(\left.\partial_{s} \partial_{t}\right|_{s, t=0}\right) E_{p}\left(f_{s, t}\right)=\int_{M}\langle J(v), w\rangle \omega
$$

for $v:=\left(\left.\partial_{s}\right|_{s, t=0}\right) f_{s, t}$ and $w:=\left(\left.\partial_{t}\right|_{s, t=0}\right) f_{s, t}$. Here $J(v)=J_{p}(v)$ is given by

1.9. $J_{p}(v)=\nabla^{*}\left\{(p-2)\|d f\|^{p-4}(\operatorname{div} v) d f+\|d f\|^{p-2} \nabla v\right\}-\|d f\|^{p-2} \operatorname{Tr} K^{N}(d f, v) d f$,

where $\operatorname{div} v$ denotes the inner product $\langle\nabla v, d f\rangle\left(=g^{i j} h_{A B} \nabla_{i} v^{A} \partial_{j} f^{B}, h\right.$ the pullback of the Riemannian metric of $N), K^{N}$ is the curvature of $N$ and $\operatorname{Tr} K^{N}(d f, v) d f$ is the trace of the $E$-valued quadratic form: $u \mapsto K^{N}(d f(u), v) d f(u)$ on the vector fields $u$ on $M$. In fact the left hand side of (1.8) equals

$$
(p-2) \int_{M}\|d f\|^{p-4}(\operatorname{div} v)(\operatorname{div} w) \omega+\int_{M}\|d f\|^{p-2}\left\{\langle\nabla v, \nabla w\rangle-\left\langle\operatorname{Tr} K^{N}(d f, v) d f, w\right\rangle\right\} \omega .
$$

The formula (1.9) agrees with [Th] (or more precisely, with the extreme right-hand side 
of (3.2) in [Th].)

In case $p=2$, the formula (1.9) reads

1.10 .

$$
J_{2}(v)=\nabla^{*} \nabla v-\operatorname{Tr} K^{N}(d f, v) d f .
$$

The operator $\nabla^{*} \nabla$ is often written $\Delta$ and called the rough Laplacian.

1.11. Definition. A $p$-harmonic map is called $p$-stable or stable iff the integral (1.8) is non-negative for every section $v=w$ of $E$, or, equivalently, the eigenvalues of the elliptic self-adjoint operator $J_{p}$ are all non-negative.

\section{The Main Results.}

Throughout this section and the rest, we assume that

2.1. The map $f: M \rightarrow N$ is isometric and totally geodesic (but not constant).

Thus $f$ is $p$-harmonic for every $p \geqq 2$ and $f$ is a minimal immersion. Recall that $f$ is minimal iff $f$ satisfies $\int_{M}(\operatorname{div} v) \omega=0$ for every section $v$ of $E$.

The tangent bundle $T M$ may be identified with a subbundle of $E$ under (2.1); hence every section $v$ of $E$ is decomposed into the sum of the tangential component $v_{T}$ and the normal one $v_{N} ; v=v_{T}+v_{N}$.

2.2. Proposition. One has $J_{p}(v)_{T}=J_{p}\left(v_{T}\right)$ and $J_{p}(v)_{N}=J_{p}\left(v_{N}\right)$ for every section $v$ of $E$; that is, $J_{p}\left(v_{T}\right)$ and $J_{p}\left(v_{N}\right)$ are the tangential and the normal components of $J_{p}(v)$ respectively.

Proof. Since $\|d f\|$ is a constant by (2.1) and (1.6), the Jacobi operator in (1.9) may be written as

2.3. $\left(\|d f\|^{p-4}\right)^{-1} J_{p}(v)=\nabla^{*}\left\{(p-2)(\operatorname{div} v) d f+\|d f\|^{2} \nabla v\right\}-\|d f\|^{2} \operatorname{Tr} K^{N}(d f, v) d f$.

We look at the right hand side and observe

$$
\operatorname{div} v=\langle\nabla v, d f\rangle=\left\langle(\nabla v)_{T}, d f\right\rangle=\left\langle\nabla v_{T}, d f\right\rangle ;
$$

2.5. $\nabla *\left\{\left(\operatorname{div} v_{T}\right) d f\right\}=-d f\left(\operatorname{grad}\left(\operatorname{div} v_{T}\right)\right)=-\operatorname{grad}\left(\operatorname{div} v_{T}\right)$, where $\operatorname{grad} \phi$ denotes the dual vector field to the 1-form $d \phi$ for the function $\phi$ on $M$;

2.6. $\quad \nabla^{*} \nabla v=\Delta v \quad$ with $(\Delta v)_{T}=\Delta v_{T}$ and $(\Delta v)_{N}=\Delta v_{N} \quad$ by (2.1);

2.7. $\operatorname{Tr} K^{N}\left(d f, v_{T}\right) d f$ is tangential and equals $\operatorname{Tr} K^{M}\left(d f, v_{T}\right) d f ; \quad$ and

2.8. $\operatorname{Tr} K^{N}\left(d f, v_{N}\right) d f$ is normal by $\left\langle K^{N}\left(d f, v_{N}\right) d f, u\right\rangle=\left\langle K^{N}(d f, u) d f, v_{N}\right\rangle$ $=\left\langle K^{M}(d f, u) d f, v_{N}\right\rangle=0$ for every tangential vector field $u$.

The proposition is proven. 
2.9. COROLLARY. The tangential and the normal components of the Jacobi operator are

2.9a.

$$
\begin{aligned}
\left(\|d f\|^{p-4}\right)^{-1} J_{p}\left(v_{T}\right) & =-(p-2) \operatorname{grad}\left(\operatorname{div} v_{T}\right)+\|d f\|^{2} J_{2}\left(v_{T}\right) \\
& =-(p-2) \operatorname{grad}\left(\operatorname{div} v_{T}\right)+\|d f\|^{2}\left(\Delta v_{T}-R^{M}\left(v_{T}\right)\right),
\end{aligned}
$$

where $R^{M}\left(v_{T}\right)=\operatorname{Tr} K^{M}\left(d f, v_{T}\right) d f$ is the Ricci transform of $v_{T}$, and

2.9b. $\left(\|d f\|^{p-4}\right)^{-1} J_{p}\left(v_{N}\right)=\|d f\|^{2} J_{\min }\left(v_{N}\right)=\|d f\|^{2}\left(\Delta v_{N}-\operatorname{Tr} K^{N}\left(d f, v_{N}\right) d f\right)$, where $J_{\min }$ is the Jacobi operator for the minimal immersion $f$.

2.10. Recall (1.11) a p-harmonic map $f$ is unstable or p-unstable iff the second variation $\left(J_{p}(v), v\right)=\int_{M}\left\langle J_{p}(v), v\right\rangle \omega$ is negative for some section $v$ of $E=f^{*} T N$.

2.11. Proposition. Under the assumption (2.1), $f$ is p-stable if and only if the identity map $1_{M}$ of $M$ is p-stable and $f$ is stable as a minimal immersion.

Proof. This is obvious from (2.9), (2.9a) and (2.9b), since $\|d f\|$ is a positive constant; technically, the proof goes like that of Lemma 7.1 of [OU] (for the case $p=2)$.

2.12. THEOREM. Let $f: M \rightarrow N$ be a non-constant $C^{\infty}$-map of a compact connected Riemannian manifold $M$ into another. Assume that $f$ is isometric and totally geodesic immersion (i.e. we assume 2.1). Then $f$ is p-stable for a sufficiently large $p$ (say, $p \geqq 2+\operatorname{dim} M$ ) if and only if $f$ is stable as a minimal immersion.

Proof. The "only if" part follows from Proposition 2.11. Thus we assume stability of $f$ as a minimal immersion. In view of (2.11), we have to show that the identity map is $p$-stable for a sufficiently large $p$. We work on $\left(J_{p}(v), v\right)$ for the sections $v=v_{T}$ of the tangent bundle $T M \subset E$. Recall a formula of K. Yano (4.4 in [Y], and quoted in [Sm]):

2.12a.

$$
\left(J_{2}(v), v\right)=\int_{M}\left\{\frac{1}{2}\left\|L_{v} g\right\|^{2}-(\operatorname{div} v)^{2}\right\} \omega,
$$

where $L_{v} g$ is the Lie derivative of the metric $g$. Then, writing $m$ for $\|d f\|^{2}=\operatorname{dim} M$, one obtains from (2.9a) and (2.12a)

$$
\begin{aligned}
& \left(m^{2-p / 2} J_{p}(v), v\right)=\int_{M}\left\langle m^{2-p / 2} J_{p}(v), v\right\rangle \omega=\left(-(p-2) \operatorname{grad}(\operatorname{div} v)+m J_{2}(v), v\right) \\
& =(p-2)(\operatorname{div} v, \operatorname{div} v)+m \int_{M}\left\{\frac{1}{2}\left\|L_{v} g\right\|^{2}-(\operatorname{div} v)^{2}\right\} \omega \\
& =(p-2-m)(\operatorname{div} v, \operatorname{div} v)+\frac{1}{2} m \int_{M}\left\|L_{v} g\right\|^{2} \omega \geqq(p-2-m)(\operatorname{div} v, \operatorname{div} v),
\end{aligned}
$$


which is nonnegative for every $v$ provided $p \geqq 2+m$.

2.12b. ACKNOWLeDGement. We owe a certain improvement of the proof to Prof. Ohnita.

2.13. THEOREM. Assume furthermore that the domain $M$ is an Einstein manifold (so that $R^{M}$ is a scalar, $c$, times the identity or the Ricci tensor equals $\mathrm{cg}$ ), in the setting of the previous Theorem 2.12. Then $f: M \rightarrow N$ is p-stable if and only if $p$ satisfies

2.14 .

$$
(p-2+\operatorname{dim} M) \lambda_{1}-2 c \operatorname{dim} M \geqq 0,
$$

where $\lambda_{1}$ is the smallest positive eigenvalue of the Laplacian $\Delta$ operating on functions and $c \operatorname{dim} M$ is the scalar curvature as mentioned; thus the estimate (2.14) is sharp.

Proof. This is easily checked by estimating $\left\langle J_{p}(\operatorname{grad} \phi), \operatorname{grad} \phi\right\rangle, \Delta \phi=\lambda_{1} \phi$. The Hodge-Kodaira theory says that every vector field is a unique sum $w+\operatorname{grad} \phi$ of a vector field $w$ with $\operatorname{div} w=0$ and a gradient for some function $\phi$; here orientability is irrelevant. Moreover the kernel Ker(div) is the orthogonal complement to the space of the gradients of the functions in the space of all the vector fields with respect to the $L^{2}$-norm. The Laplacian $\Delta+R^{M}$ (acting on the vector fields) preserves this decomposition, stabilizing these subspaces; recall $\Delta$ denotes the rough Laplacian. Under the Einstein condition in (2.13), $J_{p}$ also stabilizes them, since $R^{M}$ is then a scalar multiple of the identity; call the scalar $c$. Hence we may work on the vector fields $w$ with $\operatorname{div} w=0$ and the gradients $\operatorname{grad} \phi$ separately; that is, we have

$$
\left(J_{p}(w+\operatorname{grad} \phi), w+\operatorname{grad} \phi\right)=\left(J_{p}(w), w\right)+\left(J_{p}(\operatorname{grad} \phi), \operatorname{grad} \phi\right) .
$$

By (2.9a) and (2.12a), we have

$$
\begin{aligned}
m^{2-p / 2}\left(J_{p}(w), w\right) & =\int_{M}\left\langle m^{2-p / 2} J_{p}(w), w\right\rangle \omega=\left(-(p-2) \operatorname{grad}(\operatorname{div} w)+m J_{2}(w), w\right) \\
& =\frac{1}{2} m \int_{M}\left\|L_{v} g\right\|^{2} \omega \geqq 0,
\end{aligned}
$$

while the same formulas give

2.14a. $\quad m^{2-p / 2}\left(J_{p}(\operatorname{grad} \phi), \operatorname{grad} \phi\right)=(p-2+m)(d \Delta \phi, d \phi)-2 m c(d \phi, d \phi) ;$

in fact

$$
\begin{aligned}
\text { LHS } & =\left(-(p-2) \operatorname{grad}(\operatorname{div} \operatorname{grad} \phi)+m J_{2}(\operatorname{grad} \phi), \operatorname{grad} \phi\right) \\
& =((p-2) d \Delta \phi, d \phi)+m\left(J_{2}(\operatorname{grad} \phi), \operatorname{grad} \phi\right) \\
& =(p-2)(d \Delta \phi, d \phi)+m\left(\left(\Delta-R^{M}\right)(\operatorname{grad} \phi), \operatorname{grad} \phi\right) \\
& =(p-2)(d \Delta \phi, d \phi)+m(d \Delta \phi, d \phi)-2 m\left(R^{M}(\operatorname{grad} \phi), \operatorname{grad} \phi\right)
\end{aligned}
$$




$$
\begin{aligned}
& =(p-2+m)(d \Delta \phi, d \phi)-2 m\left(R^{M}(\operatorname{grad} \phi), \operatorname{grad} \phi\right) \\
& =(p-2+m)(d \Delta \phi, d \phi)-2 m c(d \phi, d \phi),
\end{aligned}
$$

since one has $d \Delta=\left(\Delta+R^{M}\right) d$ on the functions and $(\operatorname{grad} \phi, \operatorname{grad} \phi)=(d \phi, d \phi)$. We now recall the known facts:

$$
\text { 2.14b. } \quad(d \Delta \phi, d \phi) \geqq \lambda_{1}(d \phi, d \phi) \quad \text { for every function } \phi \text { and }
$$

2.14c. there is some $\phi$ satisfying $(d \Delta \phi, d \phi)=\lambda_{1}(d \phi, d \phi)>0$.

From these and (2.14a), we conclude that $f$ is $p$-stable if and only if $(p-2+m) \lambda_{1}-$ $2 m c \geqq 0$.

We wish to add the next corollary to illustrate a sharp contrast with the harmonic maps (See [O6]).

2.15. COROllary. The identity map of any compact Riemannian manifold is stable as a p-harmonic map for a sufficiently large $p(\geqq 2+\operatorname{dim} M)$.

Furthermore (2.14) and the proof of (2.12) yield

2.16. COROLlary. The index of the p-harmonic map $f$ equals that of the minimal immersion $f$ if $f$ is a map in Theorem (2.12) and $p$ is large enough $(p \geqq 2+\operatorname{dim} M)$.

\section{Applications to symmetric spaces.}

We are interested in the compact symmetric spaces; $M$ and $N$ will be ones for a nonconstant isometric immersion $f: M \rightarrow N$ throughout this section. A technical advantage of this additional assumption is that both the tangential and the normal components of the Jacobi operator has in their kernels the restrictions of Killing vector fields, which reduces the study of stability to comparison of the eigenvalues of the Casimir operators of relevant representations. Also one can explicitly calculate necessary invariants such as $\lambda_{1}$; thus, if $M$ is simple and 1-connected, the inequality (2.14) reads $(1+(p-2) / \operatorname{dim} M) \lambda_{1} \geqq 2$ (the metric being given by the Killing form) and the value of $\lambda_{1}$ is listed in [KOT]. So there are many results to which Theorem 2.12 applies directly, providing with numerous examples of $p$-stable mappings; $(2.13)$ is still easily applicable, although $M$ may not quite be an Einstein manifold (but a local Riemann product of Einstein manifolds). We will quote some of them, (3.1) through (3.10). We call $f$ min-stable iff $f$ is stable as a minimal immersion. $M$ is called min-stable iff $f$ is a min-stable embedding.

3.1. Assume $f$ is the inclusion of a symmetric $R$-space $M$ into the Kähler symmetric space $N=M^{c}$, which one might take as the complexification of $M$. Then $f$ is min-stable if and only if $M$ is 1-connected [T37]. 
3.2. M. Takeuchi [T40] determined min-stability of $f$ when $f$ is the inclusion of a totally complex subspace $M$ into the quaternionic symmetric space $N=M^{\boldsymbol{H}}$, $2 \operatorname{dim} M=\operatorname{dim} N$, and $N$ is a classical space. The exceptional cases have been worked out by Sumi [Su]. Here are her results (without explanation of symbols; see [N]). In case $N=$ EII, then $\mathrm{CI}(4)^{\%}$ and DIII(5) are min-stable but $S^{2} \cdot G_{3}\left(C^{6}\right)$ is not, the symbol $\%$ denoting the bottom or the adjoint space [H]. If $N$ is EVI, then $G_{4}\left(C^{8}\right) \%$ and EIII are min-stable but $S^{2} \cdot \operatorname{DIII}(6)$ is not. If $N$ is EIX, then DIII(8)\% is min-stable but $S^{2} \cdot$ EVII is not. Finally there is no min-stable totally complex subspace in FI or GI.

3.4. Ohnita [O12] proved that the Helgason spheres are min-stable and determined min-stability of totally geodesic subspaces in case $N$ has rank 1 .

3.5. Mashimo [M] determined min-stability of the Cartan embedding $f: M=G / K \rightarrow G$ when $K$ is the fixed point set of the involution of $G$ which is simple. Thus $M$ is unstable if and only if $1^{\circ} M$ is Kählerian, $2^{\circ} M$ is $\mathrm{AI}(n)$ with $G=\mathrm{SU}(n)$, $n \geqq 3,3^{\circ} \mathrm{AI}(4 m+2)$ with $G=\operatorname{SU}(4 m+2) /\{ \pm 1\}, m \geqq 1,4^{\circ} M=G_{3}^{\circ}\left(R^{n}\right)$ with $G=\operatorname{Spin}(n)$, $n \geqq 7$, or $5^{\circ} M=\mathrm{GI}$.

3.6. Mashimo and Tasaki [MT] studied the case of a subgroup $M$ of a simple group $N$. They proved, among others, that (i) $M$ is min-stable if the subgroup has the Dynkin-index $=1$; and (ii) the converse is true in case $M$ is $\mathrm{SU}(2)$.

3.7. Tasaki ([Ts] and a preceding paper) determined the quaternionic subspaces of the quaternionic Kähler symmetric spaces and proved that those subspaces are all min-stable (and even homologically minimal !).

3.8. Mashimo and Tasaki [MT2] determined min-stability of closed subgroups $G$ of maximal rank in a compact simple group $U$. More precisely, (i) $G$ is min-stable if the maximal tori $T$ are; and (ii) $T$ is not min-stable if and only if $U$ is $\operatorname{SU}(n)$, $\operatorname{Spin}(7)$, $\operatorname{Sp}(n)$ or $\mathbf{G}_{2}$. (They also showed that $G$ is $R$-homologous to zero, provided $\operatorname{dim} G<\operatorname{dim} U$.)

3.9. Ohnita [O6] determined all the symmetric spaces $M$ whose $1_{M}$ are unstable.

3.10. M. Sumi [Su] has determined min-stability of the fundamental building blocks of $M$, that is, the polars $M^{+}$and the meridians $M^{-}$of $M$. A polar $M^{+}=M^{+}(p)$ is any connected component of the fixed point set $F\left(s_{o}, M\right)$ of the symmetry $s_{o}$ at a point $o, p \in M^{+}(p)$, and the meridian $M^{-}(p)$ is "the orthogonal complement" to the polar $M^{+}(p)$; thus $M^{-}(p)$ is the component of $F\left(s_{o} \circ s_{p}, M\right)$ through $p$ (See [N]). A consequence is that $M^{+}$is $\boldsymbol{R}$-homologous to zero if $M^{+}$is min-unstable, and similarly for $M^{-}$. Also she has studied min-stability of totally geodesic spheres in $M$, all of which are known [NS].

Added in proof. One can improve the estimate in 2.12 , to the effect that the identity map $1_{M}$ is $p$-stable if $p \geqq \operatorname{dim} M$. (Note that this estimate is sharp, since $1_{M}$ is not $p$-stable 
for $p<\operatorname{dim} M$ by 2.13.) For the proof one has only to see that the Cauchy-Schwarz inequality applied to the symmetric tensor $L_{v} g$ implies $4(\operatorname{div} v)^{2} \leqq n\left\|L_{v} g\right\|^{2}$; thus one has $\left(J_{2}(v), v\right) \geqq(2 / n-1)(\operatorname{div} v, \operatorname{div} v)$. Hence one concludes

$$
n^{2-p / 2}\left(J_{p}(v), v\right) \geqq(p-n)(\operatorname{div} v, \operatorname{div} v) .
$$

\section{Bibliography}

[EL] J. Eells and L. Lemaire, Another report on harmonic maps, Bull. London Math. Soc., 20 (1988), $385-524$.

[H] S. Helgason, Differential Geometry, Lie Groups, and Symmetric Spaces, Academic Press (1978).

[KOT] S. KobaYASh, Y. OHNIta and M. TAKeUchi, On instability of Yang-Mills connections, Math. Z., 193 (1986), 165-189.

[M] K. Mashimo, On the stability of Cartan embeddings of compact symmetric spaces, Preprint.

[MT] K. MASHIMO and H. TASAKI, Stability of closed Lie subgroups in compact Lie groups, Kodai Math. J., 13 (1990), 181-203.

[MT2] K. Mashimo and H. TASAKI, Stability of maximal tori in compact Lie groups, Algebras Groups Geom., 7 (1990), 114-126.

[N] T. Nagano, The involutions of compact symmetric spaces, Tokyo J. Math., 11 (1988), 57-79.

[NS] T. NAgano and M. Sumi, The spheres in symmetric spaces, Hokkaido Math. J., 20 (1991), $331-$ 352.

[O6] Y. OHNITA, Stability of harmonic maps and standard minimal immersions, Tôhoku Math. J., 38 (1986), 259-267.

[O12] Y. OHNITA, On stability of minimal submanifolds in compact symmetric spaces, Compositio Math., 64 (1987), 157-189.

[OU] Y. OHNITA and S. UdAGAWA, Stability, complex-analyticity and constance of pluriharmonic maps from compact Kaehler manifolds, Math. Z., 205 (1990), 629-644.

[Sm] R. T. Smith, The second variation formula for harmonic mappings, Proc. Amer. Math. Soc., 47 (1975), 229-236.

[Su] M. Sumi, Stability of minimal submanifolds in symmetric sapces, in preparation.

[T37] M. TAKEUCHI, Stability of certain minimal submanifolds of compact hermitian symmetric spaces, Tôhoku Math. J., 36 (1984), 293-314.

[T40] M. TAKEUCH, Totally complex submanifolds of quaternionic symmetric spaces, Japan. J. Math., 12 (1986), 161-189.

[Th] H. TAkeUCHI, Stability and Liouville theorems of P-harmonic maps, Japan. J. Math., 17 (1991), 317-332.

[Ts] H. TASAKI, Quaternionic submanifolds in quaternionic symmetric spaces, Tôhoku Math. J., 38 (1986), 513-538.

[U] K. UHLenbeck, Harmonic maps; a direct method in the calculus of variations, Bull. Amer. Math. Soc., 76 (1970), 1082-1087.

[Y] K. Yano, On harmonic and Killing vector fields, Ann. of Math., 55 (1952), 38-45.

Present Address:

Department of Mathematics, SOPHia University

TOKYO 102, JAPAN 